\title{
Charge-Transfer Dynamics in Azobenzene Alkanethiolate Self-Assembled Monolayers on Gold
}

\author{
Cornelius Gahl, Roland Schmidt, Daniel Brete, Stephanie Paarmann, Martin \\ Weinelt \\ Fachbereich Physik, Freie Universität Berlin, Arnimallee 14, 14195 Berlin, Germany
}

\begin{abstract}
We have studied the charge transfer dynamics in azobenzene-functionalized alkanethiolate self-assembled monolayers. We compare the core-hole clock technique, i.e., resonant vs. non-resonant contributions in the azobenzene autoionization of the $\mathrm{C} 1 s-\pi^{*}$ core exciton, with the lifetime of a molecular resonance determined by two-photon photoemission spectroscopy using femtosecond laser pulses. Both techniques yield comparable charge-transfer times of $80 \pm 20 \mathrm{fs}$ for a linker consisting of three $\mathrm{CH}_{2}$ groups and one oxygen unit. Thus the quenching of the excitation is about one order of magnitude faster than the time required for the trans to cis isomerization of the azobenzene photoswitch in solution.

Keywords: self-assembled monolayers, charge transfer, core-hole clock, time-resolved photoemission, molecular switches

2010 MSC: 00-01, 99-00
\end{abstract}

\section{Introduction}

Photochromic molecules can serve as reproducible building blocks to optically manipulate and control surface and interface properties on the nanoscale $[1,2]$. This opens up interesting fields of application such as the operation of 5 molecular actuators and motors, the development of switchable sensors, or the biasing of charge transport across organic devices [3, 4]. Given the impressive

*Corresponding authors: C. Gahl (c.gahl@fu-berlin.de) and M. Weinelt (weinelt@physik.fuberlin.de)

Preprint submitted to Surface Science

August 19, 2015 
examples in biology and the large variety of photochromic molecules in solution, a major challenge is to ensure that the molecular switch maintains its functionality when adsorbed on a surface. On the one hand excitations in the substrate can lead to a quenching of the photoexcited state [5]. Therefore the coupling between molecular switch and substrate excitations must be controlled by spacer groups. On the other hand, steric and excitonic interactions among the chromophores in the adsorbate layer can impede efficient switching [6]. The latter problem can be overcome introducing large platforms or lateral spacers ${ }_{15}[5,7,8]$.

One of the best investigated molecular linker systems comprises the class of alkanethiols, which form self-assembled monolayers (SAMs) on gold substrates $[9,10,11,12]$. We employ SAMs to form well-ordered surface ensembles of azobenzene-functionalized molecules as well as to effectively decouple the photoswitch from the substrate. As sketched in Fig. 1 the azobenzene entity is coupled to the alkane chain via an oxygen bridge in para-position. The alkyl chain binds with the sulphur head group to the $\mathrm{Au}(111)$ surface and the molecules orient preferentially upright in the SAM with an angle of $\sim 30^{\circ}$ with respect to the surface normal [6]. Different rest groups $\left(\mathrm{R}=\mathrm{H}, \mathrm{CN}, \mathrm{CF}_{3}\right)$ were used as markers for X-ray photoelectron and near-edge X-ray absorption spectroscopy (XPS and NEXAFS) studies $[6,13,14]$. In the following the molecules are referred to as $\mathrm{R}-\mathrm{Az} n$, where $n$ denotes the number of $\mathrm{CH}_{2}$ units of the alkyl linker.

In the present article azobenzene-functionalized SAMs on gold serve as a model platform to study the coupling between photoswitch and substrate. We compare two experimental methods, resonant photoemission and time-resolved two-photon photoemission, which both allow for determining the charge-transfer (CT) time of electrons excited to the lowest unoccupied molecular orbitals $(\mathrm{LUMO}+\mathrm{n})$ into the substrate. Independent of the alkyl spacer length $(n=$ $353,6,10)$ the autoionization spectra of the azobenzene moiety are dominated by spectator and participator decay channels. Following the decay of the $\mathrm{C} 1 s$ to LUMO+1,2 resonances the dominant participator-decay line shows a lower in- 

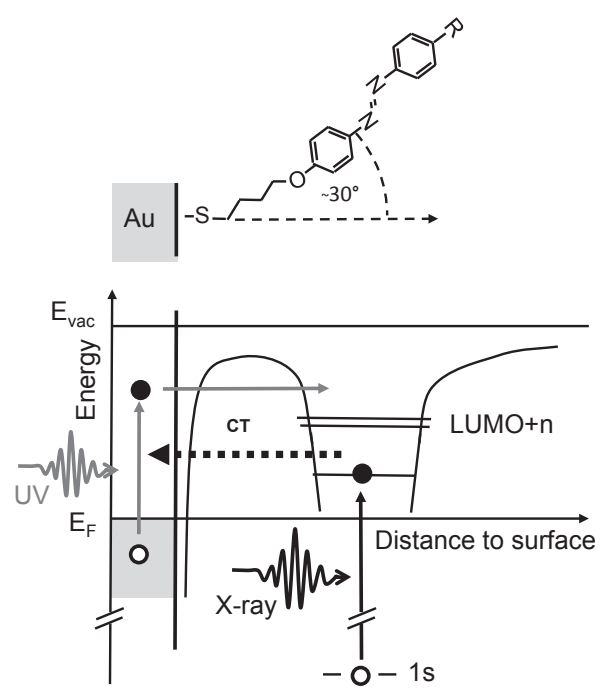

Figure 1: Alkanethiols bind with the sulphur headgroup to the $\mathrm{Au}(111)$ surface and orient preferentially upright in a self-assembled monolayer (SAM). Thereby the alkyl chain shapes a tunnel barrier, which allows for decoupling the azobenzene endgroup from the Au metal. The unoccupied molecular orbital $(\mathrm{LUMO}+\mathrm{n})$ is populated either indirectly by electrons optically excited in the gold substrate employing ultraviolet (UV) laser pulses or by resonant intramolecular excitation of a $1 s$ core electron using X-rays. The charge-transfer $(\mathrm{CT})$ time is determined by probing the excited state with a second laser pulse in a time-resolved photoemission experiment or by disentangling resonant autoionization from non-resonant Auger contributions in the core-hole decay. 
tensity for shorter chain length. Applying the core-hole clock we obtain for the $\mathrm{CF}_{3}$-Az3 SAM a charge-transfer time of $73 \pm 20 \mathrm{fs}$.

40 The 2PPE spectra of $\mathrm{H}-\mathrm{Az} 3$ show a negative ion resonance at $E-E_{F}=3.6 \mathrm{eV}$, which we attribute to a $\mathrm{LUMO}+\mathrm{n}$ of the azobenzene chromophore. The resonance has a lifetime of $80 \pm 20 \mathrm{fs}$ in good agreement with the result from the core-hole-clock technique. While the charge-transfer time is compatible with a tunnel barrier of thickness $n=4[15,16]$, it seems unaffected by the resonance 45 position.

\section{Experimental}

The various R-Azn compounds were synthesized and purified as described elsewhere $[13,17]$. Their purity has been verified by ultrahigh-performance liquid chromatography. As substrates we used $200 \mathrm{~nm}$ thick gold films on mica

${ }_{50}$ (Georg Albert PVD), which exhibit large $\mathrm{Au}(111)$ terraces. SAMs were prepared by immersing the substrate into $10^{-4}$ molar ethanolic solution for 24 hours. Afterwards the samples were copiously rinsed with pure ethanol, blown dry with argon, mounted on the sample holder and directly transferred into the ultrahigh-vacuum chambers (base pressure $\leq 2 \cdot 10^{-10} \mathrm{mbar}$ ). The preparation procedure was routinely controlled by XPS $[13,14]$.

We used two separate experimental setups for resonant and time-resolved photoemission. Both apparatus were equipped with comparable load locks and sample holders for sample transfer and cooling. To reduce X-ray beam-damage experiments were performed at a sample temperature of $\sim 120 \mathrm{~K}[18,19]$ and

60 the X-ray exposure was minimized by scanning the sample and using a fast beam shutter.

Resonant photoemission experiments were performed at the undulator beamline U41 PGM-1 of the synchrotron facility BESSY II, Helmholtz-Zentrum Berlin. We used a hemispherical analyzer (Omicron, EA125) equipped with 5 channeltrons. The total energy resolution of the measurements was $0.2 \mathrm{eV}$ as established by the width of the S2p XPS line and the Fermi level of the 
clean gold substrate. Measurements were performed in grazing incidence with an angle of $15^{\circ}$ between X-ray beam and surface plane. The analyzer chamber is rotatable around the beam axis. In this way the polarization of the incoming X-ray beam was set parallel to the surface plane and the angle of electron detection normal to the surface. This measurement geometry optimizes the ratio between the autoionization signal and the photoemission background [20]. The photon energies and the binding energy scale are referenced to the binding energy of the $\mathrm{Au} 4 f_{7 / 2}$ core level of $83.95 \mathrm{eV}$ [21].

The laser system for $2 \mathrm{PPE}$ spectroscopy consists of two optical parametric amplifiers, both pumped by a Ti:Saphire amplifier system running at a repetition rate of $300 \mathrm{kHz}$ (Coherent, RegA). In this way independently tunable visible and ultraviolet (UV) light pulses are generated with a duration of $\sim 50 \mathrm{fs}$ and pulse energies of $\sim 25 \mathrm{~nJ}$ and $3 \mathrm{~nJ}$, respectively. A translation stage is used to adjust the delay of the visible pulse. Both beams impinge nearly collinearly on the sample in the horizontal plane, which forms an angle of $45^{\circ}$ to the surface normal. Emission angle and kinetic energy of the photoemitted electrons are measured using a hemispherical electron analyzer (SPECS, Phoibos 100) equipped with a two-dimensional view-type detector.

\section{Results and Discussion}

\subsection{The core-hole clock}

One means of studying the charge transfer-time between adsorbate and substrate is the so-called core-hole clock (see, e.g. [22] and refs. therein). This method was pioneered by the groups of Dietrich Menzel in Munich and Nils Mårtensson in Uppsala initially investigating rare gas layers physisorbed on metal surfaces $[23,24]$. The method's keynote is illustrated in Fig. 2. A core-tovalence excitation starts the core-hole clock, which stops on the time scale of the core-hole lifetime $\tau_{\Gamma}$. If charge transfer between the excited valence level and the substrate occurs prior to the core-hole decay, one records an ordinary, i.e., non-resonant Auger spectrum. In contrast, if the excited electron stays on the 


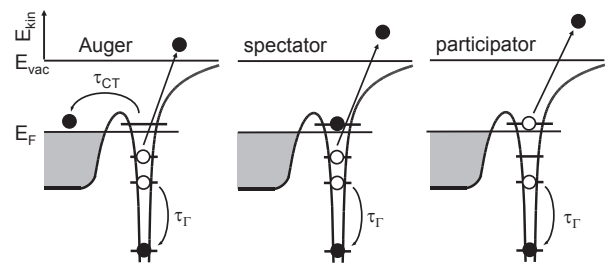

Figure 2: Schematics of the core-hole decay. After resonant excitation of a core-exciton, the charge-transfer time $\tau_{\mathrm{CT}}$ is deduced by reference to the core-hole lifetime $\tau_{\Gamma}$ comparing the intensities of non-resonant (Auger) and resonant (spectator, participator) decay channels: $\tau_{\mathrm{CT}} / \tau_{\Gamma}=\left(I_{\text {Auger }}+I_{\text {spectator }}+I_{\text {participator }}\right) / I_{\text {Auger }}$.

adsorbate it may either participate in the autoionization or additionally screen the core-hole decay. This results in either an enhancement of photoemission lines (participator decay) or a screening shift of the Auger decay spectrum to higher kinetic energies (spectator shift). Assuming exponential decay, the charge transfer time $\tau_{\mathrm{CT}}$ can be deduced from the intensities of non-resonant $I_{\text {Auger }}$ and resonant $I_{\text {participator }}+I_{\text {spectator }}$ Auger channels $\tau_{\mathrm{CT}} / \tau_{\Gamma}=\left(I_{\text {res }}+I_{\text {Auger }}\right) / I_{\text {Auger }}$ $[25,26]$. Given core-hole lifetimes of 6.6 and $5.4 \mathrm{fs}$ for the carbon and nitrogen $1 s$-levels [27, 28], charge-transfer times ranging from sub-fs to about $100 \mathrm{fs}$ can be determined. For the light elements the X-ray emission yield is below $1 \%$ and can be neglected. A major advantage of the core-hole-clock technique is its sub-monolayer sensitivity, which results from the pronounced cross section of resonant core-to-valence transitions. A challenge for larger molecules is the increasing number of Auger decay channels. In combination with vibrational excitations, they lead to complex decay spectra, which are hard to decompose.

\subsection{Core-ionized vs. core-excitonic state of azobenzene}

In the core-ionized state a core hole in the azobenzene endgroup will be screened by polarization of the SAM and the gold substrate. In the coreexcitonic state the binding energy of the resonantly excited occupied molecular level is increased mainly by the Coulomb interaction with the core hole. To observe charge transfer between adsorbate and substrate it is a prerequisite that 
the energy of the excited molecular level stays above the Fermi level in the coreexcitonic state. In other words, the energy of the NEXAFS resonance has to exceed the XP binding-energy of the core electron, which refers to $E_{F}$. In the azobenzene moiety this becomes element-specific. Figures $3 \mathrm{a}$ and $\mathrm{b}$ compare the XP and NEXAFS spectra of the chromophore's N1s and C1s core-levels (top and bottom panels) for SAMs with alkyl chain-lengths of $n=3,6$ and 10, respectively. Note that on the common energy scale the abscissae of the XP spectra are reverted so that shake-up excitations are observed on the right of the main line. Given the preferentially upright geometry of the molecules in all SAMs and the inelastic mean-free-path of the photoelectrons $[29,30]$ the C1s XP signal is dominated by the azobenzene chromophore with minor contributions from the alkyl chain.

As evident from Figs. 3a and $\mathrm{b}$ the core-hole binding-energies, i.e., the coreionized XP final states depend on the alkyl chain length. Both $\mathrm{N} 1 s$ and $\mathrm{C} 1 s$ peak maxima shift by $\sim 400 \mathrm{meV}$ to higher binding energy with increasing number of $\mathrm{CH}_{2}$ units. This shift is attributed to a final state effect as the initial state of the chromophore is comparable for all chain lengths. Image-charge screening of the core hole becomes less efficient for larger distance to the metal substrate. Therefore the binding energy increases for longer spacer length. A similar effect has already been observed in the C1s XP spectra of alkanethiolate SAMs [31] and for the N1s XP line of a cyano end group [16].

In contrast to the varying XP binding energies the $\pi^{*}$-resonances stay at constant energy independent of the alkyl chain-length. Screening of the core hole is dominated by the interaction with the electron in the unoccupied molecular orbital forming the core exciton. The resonance at the nitrogen absorption edge at $398.3 \mathrm{eV}$ is assigned to the azobenzene $\mathrm{N} 1 s$ to $\pi^{*}$ LUMO transition. Its energy is lower than the $\mathrm{N} 1 s$ XP peak maximum of $\mathrm{CF}_{3}-\mathrm{Az} 3$ at $399.4 \mathrm{eV}$. As a consequence the core exciton can solely decay via autoionization but not via transfer of the excited electron into unoccupied states of the substrate. 145 The significant broadening of the $\pi^{*}$-resonance beyond the natural linewidth of $100 \mathrm{meV}$ is therefore attributed to vibrational excitations. 

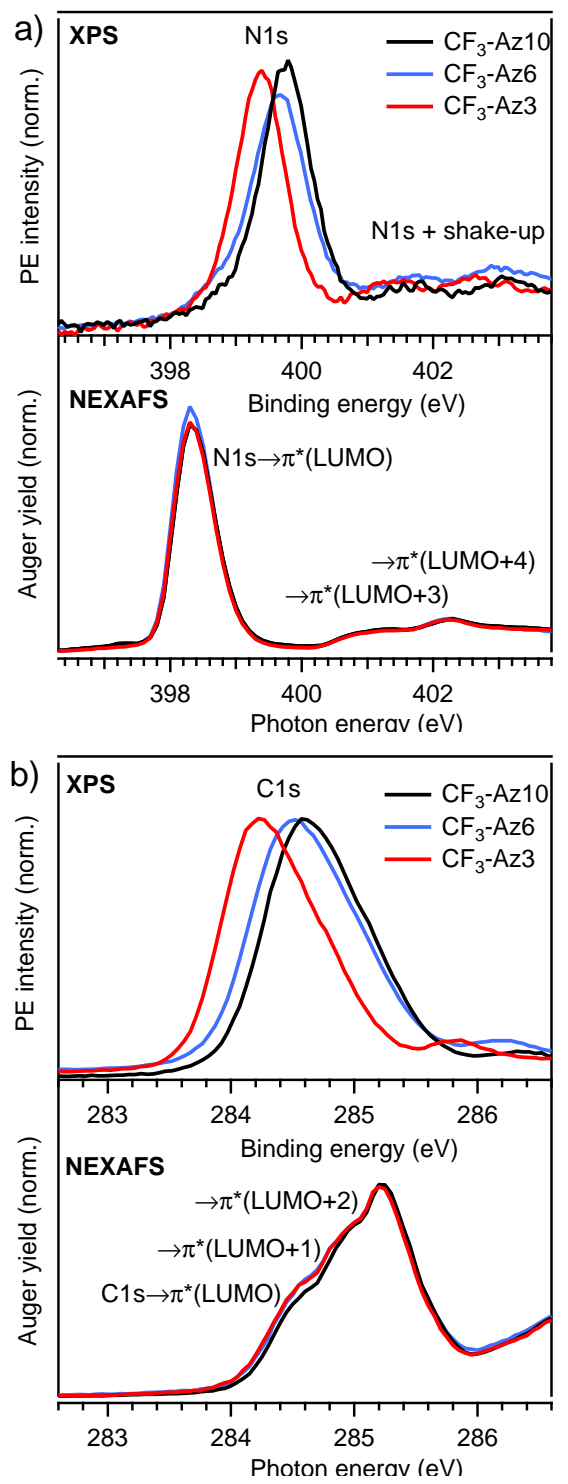

Figure 3: Comparison of core-ionized and core-excited states in XP and NEXAFS spectroscopy for $\mathrm{CF}_{3}$-Azn SAMs with alkyl chain-lengths $n=3,6$ and 10 at a) the nitrogen and b) the carbon $1 s$ edge. XP and NEXAFS spectra are plotted on a common energy scale referring to the $1 s$ binding energy and the photon energy, respectively. The XP spectra shift to higher binding energy with increasing chain length reflecting the reduced image-charge screening of the XP final state by the substrate. At the N1s edge the $\pi^{*}$-resonance is lower in energy than the XP core level. Thus the LUMO is pulled below the Fermi energy in the core-excited state and charge transfer from adsorbate to substrate can not occur. In contrast at the $\mathrm{C} 1 s$ edge charge transfer is possible out of the energeticadly higher LUMO+1 and LUMO+2 resonances. 
The situation is different at the $\mathrm{C} 1 s$ absorption edge. The first absorption line shows a multiple peak structure, which reflects contributions from the LUMO as well as the LUMO+1 and LUMO+2. A contour plot of the unoccupied orbitals can be found in Fig. 6 of Ref. [13]. The energy of the resonance maximum at $285.25 \mathrm{eV}$ is clearly above the XP peak position at $284.2 \mathrm{eV}(n=3)$. Therefore charge transfer between the azobenzene chromophore and the gold substrate can occur after resonant excitation of the $\mathrm{C} 1 s$ level.

\subsection{Charge-transfer time}

To study the charge-transfer time we concentrate in the following on the decay spectra after C1s excitation. Figure 4a shows three photoemission spectra of $\mathrm{CF}_{3}-\mathrm{Az} 3$ recorded for photon energies below, at, and above the $\mathrm{C} 1 s$ resonance maximum. The photoemission spectra have been normalized to the gold valence band intensity and are plotted on a kinetic energy scale.

The spectrum recorded below the $\pi^{*}$-resonance at $h \nu=275.0 \mathrm{eV}$ is dominated by photoemission from the gold $6 s p$ and $5 d$ valence bands, which extend over a range of $\sim 11 \mathrm{eV}$ below $E_{F}$ [32]. In addition there are minor contributions from the valence orbitals of the SAM and the C1s XP line at $262 \mathrm{eV}$ kinetic energy ionized by the second harmonic of the undulator $(2 h \nu=550.0 \mathrm{eV})$.

The spectrum recorded at a photon energy of $312.0 \mathrm{eV}$ above the ionization threshold is representative for non-resonant Auger decay. The multitude of azobenzene valence orbitals involved in the KVV decay of the $\mathrm{C} 1 s$ core hole give rise to a weakly structured $30-\mathrm{eV}$ wide Auger peak centered at a kinetic energy of $260.0 \mathrm{eV}$.

Tuning the photon energy to the resonance maximum at $285.25 \mathrm{eV}$ we resonantly excite the close lying $\mathrm{LUMO}+2$ and $\mathrm{LUMO}+3$, which extend over the outer and inner phenyl ring of the azobenzene chromophore, respectively. These core-excited states predominantly decay via resonant photoemission. The center of mass of the autoionization spectrum is at $262.0 \mathrm{eV}$ kinetic energy and the spectrum exhibits a more pronounced fine structure as compared to the nonresonant Auger decay. The higher kinetic energy is attributed to a spectator 

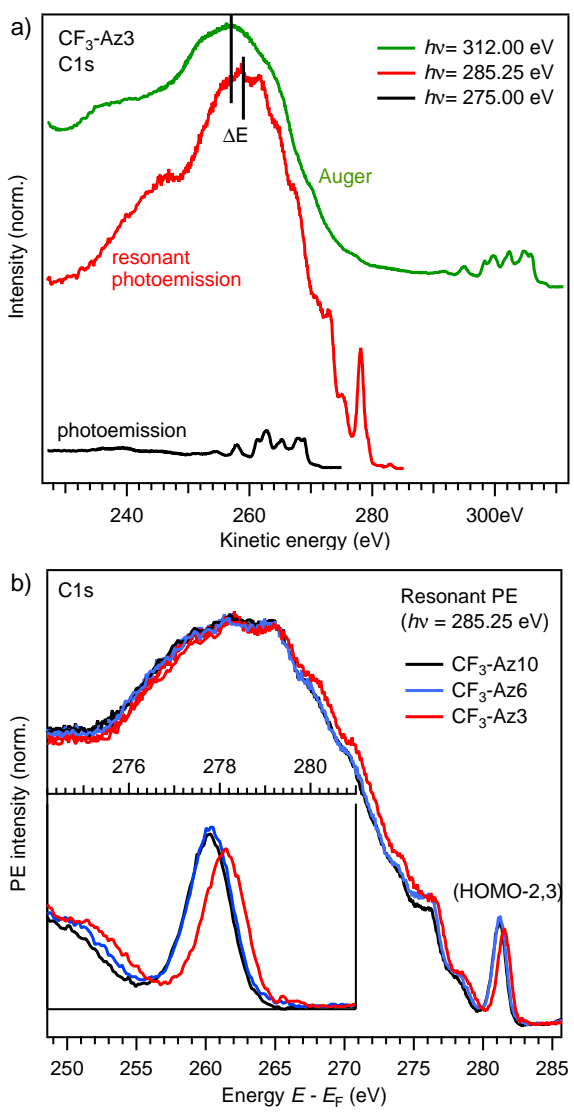

Figure 4: a) Photoemission spectra of a $\mathrm{CF}_{3}-\mathrm{Az} 3 \mathrm{SAM}$ recorded with photon energies below $(h \nu=275.0 \mathrm{eV})$, at $(h \nu=285.25 \mathrm{eV})$, and above $(h \nu=302.0 \mathrm{eV})$ the maximum of the $\mathrm{C} 1 s$ to $\pi^{*}$ LUMO $+1,2$ transitions ( $c f$. Fig $3 \mathrm{~b}$ ). Spectra have been normalized to equal intensity of the Ausp band $1 \mathrm{eV}$ below $E_{F}$. The vertical bars indicate the spectator shift. The HOMO-2,3 participator line is strongly enhanced at resonance. b) Comparison of resonant photoemission spectra of $\mathrm{CF}_{3}-\mathrm{Az} n$ for alkyl chain length of $n=3,6$ and 10. All spectra have been recorded at a photon energy of $285.25 \mathrm{eV}$ and are normalized to equal intensity after subtracting the nonresonant photoemission background. The shift of the spectra is a final state effect attributed to distinct polarization screening. As highlighted in the inset the HOMO-2,3 participator decay line has a $9 \pm 2 \%$ lower intensity for the shortest alkyl chain length $n=3$ as compared to $n=6$ and 10 
shift of $2.0 \pm 0.2 \mathrm{eV}$ and the fine structure to additional participator decay lines. The comparable width of the Auger and autoionization spectra indicates that there exist only weak contributions of non-resonant Auger decay at resonance. In other words, the charge transfer between adsorbate and substrate must be slow compared to the core-hole lifetime. The most prominent spectator line of the autoionization spectrum is observed at a kinetic energy of $278.15 \mathrm{eV}$, which corresponds to a binding energy of $E-E_{F}=2.7 \mathrm{eV}$. It is attributed to the nearly degenerate HOMO-2 and HOMO-3 of the azobenzene moiety, which extend over the inner and outer phenyl ring, respectively. The HOMO is the lone-pair orbital of the azo-bridge and thus localized at the nitrogen atoms. It is therefore not enhanced in resonant photoemission at the carbon edge. Given the small spectator shift in $\mathrm{C} 1 s$ autoionization it is very difficult to decompose the decay spectra in resonant and non-resonant contributions. However, we can extract a charge transfer time for the $\mathrm{CF}_{3}$ - $\mathrm{Az} 3 \mathrm{SAM}$ by comparing the intensity of the main participator line for the three different alkyl chain length.

Figure 4b shows the decay spectra of $\mathrm{CF}_{3}$-Azn for $n=3,6$ and 10, respectively, recorded at resonance $(h \nu=285.25 \mathrm{eV})$ after subtraction of the nonresonant photoemission background $(h \nu=275.0 \mathrm{eV})$. Prior to subtraction, the spectra have been aligned at the $\mathrm{Au} 4 f$ level and normalized with respect to the NEXAFS spectrum (Fig. 3b), which was recorded in Auger yield. This procedure yielded identical intensities of the Au $6 s p$ band close to $E_{F}$ in nonresonant and resonant photoemission. Comparable to the $\mathrm{C} 1 s$ XP spectra of Fig. $3 \mathrm{~b}$ the resonant photoemission spectra in Fig. $4 \mathrm{~b}$ shift by $400 \mathrm{meV}$ to higher kinetic energy (lower binding energy) with decreasing alkyl chain length ( $n=10$ vs. $n=3)$. The identical shift of $\mathrm{XP}$ and autoionization spectra suggests that screening of the one-hole and two-holes-one-electron valence states reached after autoionization ( $c f$. Fig. 2) is comparable to screening of the $1 s$ core hole. As already discussed, polarization screening of the excited state is dominated by the substrate's contribution and thus rather insensitive to the charge distribution in the chromophore but less effective for larger distance to the metal surface. To evaluate the charge transfer time spectra in Fig. 4b have been normalized 
to equal area. The intensity of the HOMO-2,3 participator line for $\mathrm{CF}_{3}-\mathrm{Az} 3$ is by $9 \pm 2 \%$ smaller than for the longer alkyl spacers $n=6$ and 10 (see inset the former directly probes the optical-excited photoswitch in the absence of a 
core hole.

Figure 5a shows a false color plot of a time- and energy-resolved $2 \mathrm{PPE}$

states close above the vacuum level $E_{v a c}$ [39], and we therefore refrain from evaluating this contribution further.

The 2PPE intensity at $5.7 \mathrm{eV}$ final state energy extends towards positive 

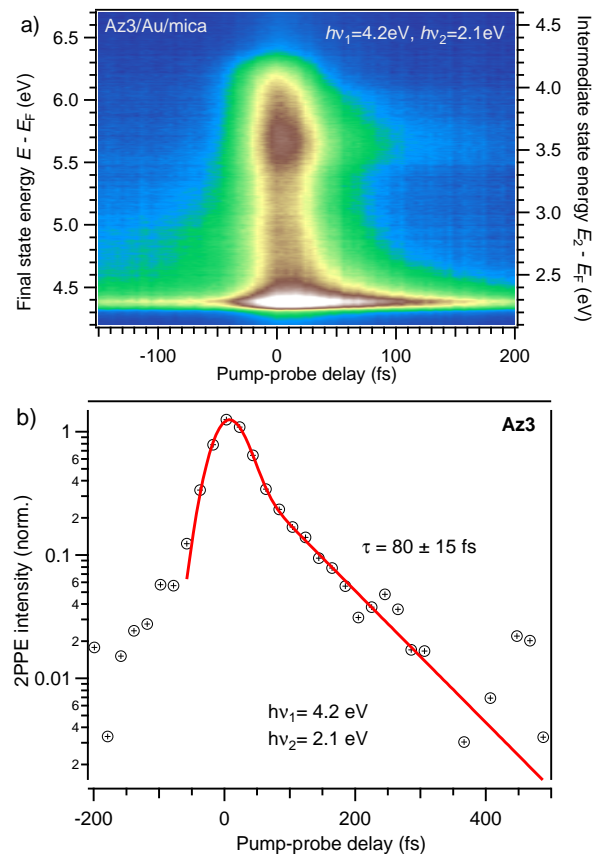

Figure 5: a) False color plot of a time- and energy-resolved 2PPE spectrum for a H-Az3 SAM. The left ordinate refers to the final state energy $E-E_{F}$. The right ordinate shows the energy of an intermediate state $E_{2}-E_{F}$ populated with the ultraviolet pulse $h \nu_{1}=4.2 \mathrm{eV}$ and ionized with visible light $h \nu_{2}=2.1 \mathrm{eV}$, which refers to positive pump-probe delay. For negative pump-probe delay $h \nu_{2}$ arrives before $h \nu_{1}$ at the sample. b) Time-resolved trace extracted in an energy range of $\pm 0.1 \mathrm{eV}$ centered at 3.6-eV intermediate state energy. The solid line is a fit to the data, describing an instantaneous signal plus an exponential decay, both convolved with a 70-fs Gaussian to account for the cross-correlation of pump and probe pulses. The finite lifetime of the intermediate state of $80 \pm 15 \mathrm{fs}$ corresponds to the pure exponential decay seen at delays $>100 \mathrm{fs}$. 
pump-probe delay. It thus stems from an intermediate state at $E_{2}-E_{F}=3.6 \mathrm{eV}$,

\subsection{Comparison of charge-transfer times}

Applying the core-hole clock, the groups of Menzel, Feulner, and Zharnikov teamed up to study the charge transfer across alkyl chains varying their length from two to four $\mathrm{CH}_{2}$-groups $[15,16]$. They used SAMs with a cyano endgroup directly coupled to the alkanethiolate and measured the charge-transfer time out of the cyano LUMO after resonant excitation of the N1s to $\pi^{*}$ transition. In the core-excited state the $\pi^{*}$-LUMO is located at an energy of $0.91-0.55 \mathrm{eV}$ above $E_{F}$ for $n=2-4$, respectively. With increasing chain length resonant decay channels dominate the Auger spectrum and the charge-transfer time from the 
cyano endgroup to the substrate increases exponentially with the alkyl chainalkanethiolate SAMs varying the chain-length between $n=12$ and 18 [40]. Electrons were photoexcited into an image-potential state located in front of the SAM surface at $3.7 \mathrm{eV}$ above $E_{F}$. The charge-transfer time shows a well-defined exponential increase from about 30 to $80 \mathrm{ps}$ at $90 \mathrm{~K}$ [40]. The corresponding

described experiments. This indicates that $\beta$ depends strongly on the resonance position with respect to the barrier heights, which is at $E-E_{F} \sim 4.0 \mathrm{eV}$ [45]. In a very recent study the authors used a decanethiolate SAM on $\mathrm{Au}(111)$ as template to adsorb and isolate ferrocene molecules [41]. They observed a in contrast to the larger participator contribution for longer chain length $\left(\mathrm{CF}_{3^{-}}\right.$ $\mathrm{Az6}$ and $\mathrm{CF}_{3}-\mathrm{Az10}$ ), which indicates a significant increase in charge-transfer time, beyond the sensitivity of the core-hole clock. 
hole lifetime. Therefore charge neutralization occurs long after the photoemission event and the binding energy is lowered by polarization screening of the photo hole. This is corroborated by the shift of the valence states and core levels in the photoemission spectra to higher kinetic energy with decreasing alkyl 355

\section{Conclusion}

Azobenzene-functionalized alkanethiolate SAMs provide a model system to study the dynamics of the deexcitation of photoswitches at a metal surface. 
Charge transfer between the azobenzene endgroup and the gold substrate across M. Mayor, M. A. Rampi, Light-powered electrical switch based on cargolifting azobenzene monolayers, Angew. Chem., Int. Ed. 47 (2008) 34073409 . 
[4] N. Crivillers, E. Orgiu, F. Reinders, M. Mayor, P. Samorì, Optical modulation of the charge injection in an organic field-effect transistor based on photochromic self-assembled-monolayer-functionalized electrodes, Adv. Mater. 23 (2011) 1447-1452.

[5] M. Wolf, P. Tegeder, Reversible molecular switching at a metal surface: A case study of tetra-tert-butyl-azobenzene on $\mathrm{Au}(111)$, Surf. Sci. 603 (2009) $1506-1517$.

[6] C. Gahl, R. Schmidt, D. Brete, E. McNellis, W. Freyer, R. Carley, K. Reuter, M. Weinelt, Structure and excitonic coupling in self-assembled monolayers of azobenzene-functionalized alkanethiols, J. Am. Chem. Soc. 132 (2010) 1831-1838.

[7] B. Baisch, D. Raffa, U. Jung, O. M. Magnussen, C. Nicolas, J. Lacour, J. Kubitschke, R. Herges, Mounting freestanding molecular functions onto surfaces: The platform approach, J. Am. Chem. Soc. 131 (2009) 442-443.

[8] T. Moldt, D. Brete, D. Przyrembel, S. Das, J. R. Goldman, P. K. Kundu, C. Gahl, R. Klajn, M. Weinelt, Tailoring the properties of surfaceimmobilized azobenzenes by monolayer dilution and surface curvature, Langmuir 31 (2015) 1048-1057.

[9] J. C. Love, L. A. Estroff, J. K. Kriebel, R. G. Nuzzo, G. M. Whitesides, Selfassembled monolayers of thiolates on metals as a form of nanotechnology, Chem. Rev. 105 (2005) 1103-1169.

[10] M. Kind, C. Wöll, Organic surfaces exposed by self-assembled organothiol monolayers: Preparation, characterization, and application, Prog. Surf. Sci. 84 (2009) 230-278.

[11] C. Vericat, M. E. Vela, G. Benitez, P. Carro, R. C. Salvarezza, Selfassembled monolayers of thiols and dithiols on gold: new challenges for a well-known system, Chem. Soc. Rev. 39 (2010) 1805-1834. 
[12] D. P. Woodruff, The interface structure of n-alkylthiolate self-assembled monolayers on coinage metal surfaces, Phys. Chem. Chem. Phys. 10 (2008) $7211-7221$.

[13] R. Schmidt, E. McNellis, W. Freyer, D. Brete, T. Gießel, C. Gahl, K. Reuter, M. Weinelt, Azobenzene-functionalized alkanethiols in selfassembled monolayers on gold, Appl. Phys. A 93 (2008) 267-275.

[14] D. Brete, D. Pryzembel, C. Eickhoff, R. Carley, W. Freyer, K. Reuter, C. Gahl, M. Weinelt, Mixed self-assembled monolayers of azobenzene photoswitches with trifluoromethyl and cyano end-groups, J. Physics: Cond. mat. 24 (2012) 394015.

[15] S. Neppl, U. Bauer, D. Menzel, P. Feulner, A. Shaporenko, M. Zharnikov, P. Kao, D. Allara, Charge transfer dynamics in self-assembled monomolecular films, Chem. Phys. Lett. 447 (2007) 227-231.

[16] P. Kao, S. Neppl, P. Feulner, D. L. Allara, M. Zharnikov, Charge transfer time in alkanethiolate self-assembled monolayers via resonant auger electron spectroscopy, J. Phys. Chem. C 114 (2010) 13766-13773.

[17] W. Freyer, D. Brete, R. Schmidt, C. Gahl, R. Carley, M. Weinelt, Switching behavior and optical absorbance of azobenzene-functionalized alkanethiols in different environments, J. Photochem. Photobiol. A Chem. 204 (2009) 102-109.

[18] P. Feulner, T. Niedermayer, K. Eberle, R. Schneider, D. Menzel, A. Baumer, E. Schmich, A. Shaporenko, Y. Tai, M. Zharnikov, Strong temperature dependence of irradiation effects in organic layers, Phys. Rev. Lett. 93 (2004) 178302.

[19] P. Feulner, T. Niedermayer, K. Eberle, R. Schneider, D. Menzel, A. Baumer, E. Schmich, A. Shaporenko, Y. Tai, M. Zharnikov, Strong temperature dependence of irradiation effects in organic layers, Surf. Sci. 593 (2005) 252-255. 
[20] M. Weinelt, A. Nilsson, M. Magnuson, T. Wiell, O. Karis, M. S. N. Mårtensson, J. Stöhr, Resonant photoemission in Ni metal at the 2p edges: resonant raman and interference effects, Phys. Rev. Lett. 78 (1997) 967-970.

[21] M. P. Seah, Summary of ISO/TC 201, standard VII ISO 15472, 2001 surface chemical analysis x-ray photoelectron spectrometers-calibration of energy scales, Surf. Interface Anal. 31 (2001) 721.

[22] W. Wurth, A. Föhlisch, Electron transfer investigated by x-ray spectroscopy, in: U. Bovensiepen, H. Petek, M. Wolf (Eds.), Dynamics at Solid State Surfaces and Interfaces, Vol. 1, Wiley-VCH, Weinheim, 2010, pp.

[23] W. Wurth, P. Feulner, D. Menzel, Resonant excitation and decay of adsorbate core holes, Phys. Scripta. T41 (1992) 213-216.

[24] O. Björneholm, A. Sandell, A. Nilsson, N. Mårtensson, J. N. Andersen, Autoionization of adsorbates, Phys. Scripta. T41 (1992) 217-225.

[25] O. Björneholm, A. Nilsson, A. Sandell, B. Hernnäs, N. Mårtensson, Determination of time scales for charge-transfer screening in physisorbed molecules, Phys. Rev. Lett. 68 (1992) 1892-1895.

[26] W. Wurth, G. Rocker, P. Feulner, R. Scheuerer, L. Zhu, D. Menzel, Core excitation and deexcitation in argon multilayers: Surface- and bulk-specific transitions and autoionization versus auger decay, Phys. Rev. B 47 (1993) $6697-6704$.

[27] T. X. Carroll, J. Hahne, T. D. Thomas, L. J. Sæthre, N. Berrah, J. Bozek, E. Kukk, Carbon $1 s$ core-hole lifetime in $\mathrm{CO}_{2}$, Phys. Rev. A 61 (2000) 042503.

[28] N. Saito, A. Hempelmann, F. Heiser, O. Hemmers, K. Wieliczek, J. Viefhaus, U. Becker, Lifetime effects on the dissociation of core-excited $\mathrm{n}_{2}$ and co molecules, Phys. Rev. A 61 (2000) 022709. 
[29] C. L. A. Lamont, J. Wilkes, Attenuation length of electrons in selfassembled monolayers of n-alkanethiols on gold, Langmuir 15 (1999) 20372042.

[30] M. Zharnikov, S. Frey, K. Heister, M. Grunze, An extension of the mean free path approach to x-ray absorption spectroscopy, J. Elec. Spec. and Rel. Phen. 124 (2002) 15-24.

[31] K. Heister, L. S. Johansson, M. Grunze, M. Zharnikov, A detailed analysis of the $\mathrm{c}$ 1s photoemission of n-alkanethiolate films on noble metal substrates, Surf. Sci. 529 (2003) 36-46.

[32] D. A. Shirley, High-resolution x-ray photoemission spectrum of the valence bands of gold, Phys. Rev. B 5 (1972) 4709-4714.

[33] H. Petek, S. Ogawa, Femtosecond time-resolved two-photon photoemission studies of electron dynamics in metals, Prog. Surf. Sci. 56 (1997) 239-346.

[34] M. Weinelt, Time-resolved two-photon photoemission from metal surfaces, J. Phys.: Condens. Matter 14 (2002) R1099.

[35] T. Hertel, E. Knoesel, M. Wolf, G. Ertl, Ultrafast electron dynamics at $\mathrm{cu}(111)$ : Response of an electron gas to optical excitation, Phys. Rev. Lett. 76 (1996) 535-538.

[36] I. L. Shumay, U. Höfer, Ch. Reuß, U. Thomann, W. Wallauer, Th. Fauster, Lifetimes of image-potential states on $\mathrm{Cu}(100)$ and $\mathrm{Ag}(100)$ measured by femtosecond time-resolved two-photon photoemission, Phys. Rev. B 58 (1998) 13974-13981.

[37] S. Hagen, P. Kate, F. Leyssner, D. Nandi, M. Wolf, P. Tegeder, Excitation mechanism in the photoisomerization of a surface-bound azobenzene derivative: Role of the metallic substrate, J. Chem. Phys. 129 (2008) 164102.

[38] J. Cao, Y. Gao, H. E. Elsayed-Ali, R. J. D. Miller, D. A. Mantell, Femtosecond photoemission study of ultrafast electron dynamics in single-crystal Au(111) films, Phys. Rev. B 58 (1998) 10948-10952. 
[39] S. Hagen, F. Leyssner, D. Nandi, M. Wolf, P. Tegeder, Reversible switching of tetra-tert-butyl-azobenzene on a $\mathrm{Au}(111)$ surface induced by light and thermal activation, Chem. Phys. Lett. 444 (2007) 85-90.

[40] M. Shibuta, N. Hirata, R. Matsui, T. Eguchi, A. Nakajima, Charge separation at the molecular monolayer surface: Observation and control of the dynamics, J. Phys. Chem. Lett. 3 (2012) 981-985.

[41] M. Shibuta, N. Hirata, T. Eguchi, A. Nakajima, Probing of an adsorbatespecific excited state on an organic insulating surface by two-photon photoemission spectroscopy, J. Am. Chem. Soc. 136 (2014) 1825-1831.

[42] J. Tomfohr, O. F. Sankey, Theoretical analysis of electron transport through organic molecules, J. Chem. Phys. 120 (2004) 1542-1554.

[43] E. A. Weiss, J. K. Kriebel, M.-A. Rampi, G. M. Whitesides, The study of charge transport through organic thin films: mechanism, tools and applications, Phil. Trans. R. Soc. A 365 (2007) 1509-1537.

[44] B. Kim, J. M. Beebe, Y. Jun, X.-Y. Zhu, C. D. Frisbie, Correlation between homo alignment and contact resistance in molecular junctions: Aromatic thiols versus aromatic isocyanide, J. Am. Chem. Soc. 128 (2006) 4970-4971.

[45] M. Muntwiler, C. D. Lindstrom, X. Y. Zhu, Delocalized electron resonance at the alkanethiolate self-assembled monolayer/Au(111) interface, J. Chem. Phys. 124 (2006) 081104.

[46] A. Föhlisch, S. Vijayalakshmi, A. Pietzsch, M. Nagasono, W. Wurth, P. Kirchmann, P. Loukakos, U. Bovensiepen, M. Wolf, M. Tchaplyguine, F. Hennies, Charge transfer dynamics in molecular solids and adsorbates driven by local and non-local excitations, Surf. Sci. 606 (2012) 881-885.

${ }_{520}$ [47] T. Nägele, R. Hoche, W. Zinth, J. Wachtveitl, Femtosecond photoisomerization of cis-azobenzene, Chem. Phys. Lett. 272 (1997) 489-495. 
[48] H. Satzger, C. Root, M. Braun, Excited-state dynamics of trans- and cisazobenzene after UV excitation in the $\pi \pi^{\star}$ band, J. Phys. Chem. A 108 (2004) 6265-6271.

${ }_{525}$ [49] I. Conti, M. Garavelli, G. Orlandi, The different photoisomerization efficiency of azobenzene in the lowest $\mathrm{n} \pi^{*}$ and $\pi \pi^{*}$ singlets: The role of a phantom state, J. Am. Chem. Soc. 130 (2008) 5216-5230. 